This document is confidential and is proprietary to the American Chemical Society and its authors. Do not copy or disclose without written permission. If you have received this item in error, notify the sender and delete all copies.

\title{
Prediction errors of molecular machine learning models lower than hybrid DFT error
}

\begin{tabular}{|r|l|}
\hline Journal: & Journal of Chemical Theory and Computation \\
\hline Manuscript ID & ct-2017-00577j.R1 \\
\hline Manuscript Type: & Article \\
\hline Date Submitted by the Author: & 19-Sep-2017 \\
\hline Complete List of Authors: & $\begin{array}{l}\text { Faber, Felix; University of Basel } \\
\text { Hutchison, Luke; Google } \\
\text { Huang, Bing; University of Basel } \\
\text { Gilmer, Justin; Google } \\
\text { Schoenholz, Samuel; Google } \\
\text { Dahl, George; Google } \\
\text { Vinyals, Oriol; Google } \\
\text { Kearnes, Steven; Google } \\
\text { Riley, Patrick; Google } \\
\text { von Lilienfeld, O. Anatole; University of Basel }\end{array}$ \\
\hline
\end{tabular}

\section{SCHOLARONE \\ Manuscripts}




\section{Abstract}

We investigate the impact of choosing regressors and molecular representations for the construction of fast machine learning (ML) models of thirteen electronic ground-state properties of organic molecules. The performance of each regressor/representation/property combination is assessed using learning curves which report outof-sample errors as a function of training set size with up to $\sim 118 \mathrm{k}$ distinct molecules. Molecular structures and properties at hybrid density functional theory (DFT) level of theory come from the QM9 database [Ramakrishnan et al, Scientific Data 1140022 (2014)] and include enthalpies and free energies of atomization , $\mathrm{HOMO} / \mathrm{LUMO}$ energies and gap, dipole moment, polarizability, zero point vibrational energy, heat capacity and the highest fundamental vibrational frequency. Various molecular representations have been studied (Coulomb matrix, bag of bonds, BAML and ECFP4, molecular graphs $(\mathrm{MG})$ ), as well as newly developed distribution based variants including histograms of distances (HD), and angles (HDA/MARAD), and dihedrals (HDAD). Regressors include linear models (Bayesian ridge regression (BR) and linear regression with elastic net regu- larization $(\mathrm{EN}))$, random forest $(\mathrm{RF})$, kernel ridge regression (KRR) and two types of neural networks, graph convolutions (GC) and gated graph networks (GG). Out-of sample errors are strongly dependent on the choice of representation and regressor and molecular property. Electronic properties are typically best accounted for by MG and GC, while energetic properties are better described by HDAD and KRR. The specific combinations with the lowest out-of-sample errors in the $\sim 118 \mathrm{k}$ training set size limit are (free) energies and enthalpies of atomization (HDAD/KRR), HOMO/LUMO eigenvalue and gap (MG/GC), dipole moment (MG/GC), static polarizability (MG/GG), zero point vibrational energy (HDAD/KRR), heat capacity at room temperature (HDAD/KRR), and highest fundamental vibrational frequency (BAML/RF). We present numerical evidence that ML model predictions deviate from DFT (B3LYP) less than DFT (B3LYP) deviates from experiment for all properties. Furthermore, out-of-sample prediction errors with respect to hybrid DFT reference are on par with, or close to, chemical accuracy. The results suggest that ML models could be more accurate than hybrid DFT if explicitly electron correlated quantum (or experimental) data was available. 


\section{Introduction}

Due to its favorable trade-off between accuracy and computational cost, Density Functional Theory (DFT) $)^{1,2}$ is the workhorse of quantum chemistry ${ }^{3}$ - despite its well known shortcomings regarding spin-states, van der Waals interactions, and chemical reactions. ${ }^{4,5}$ Failures to predict reaction profiles are particularly worrisome, ${ }^{6}$ and recent analysis casts even more doubts on the usefulness of DFT functionals obtained through parameter fitting. ${ }^{7}$ The prospect of universal and computationally much more efficient machine learning (ML) models, trained on data from experiments or generated at higher levels of electronic structure theory such as post-Hartree Fock or quantum Monte Carlo (e.g. exemplified in Ref. ${ }^{8}$ ), therefore represents an appealing alternative strategy. Not surprisingly, a lot of recent effort has been devoted to developing ever more accurate ML models of properties of molecular and condensed phase systems.

Several ML studies have already been published using a data set called QM9, ${ }^{9}$ consisting of molecular quantum properties for the $\sim 134 \mathrm{k}$ smallest organic molecules containing up to 9 heavy atoms (C, O, N, or F; not counting $\mathrm{H}$ ) in the GDB-17 universe. ${ }^{10}$ Some of these studies have developed or used representations we consider in this work, such as BAML (Bonds, angles, machine learning), ${ }^{11}$ bag of bonds (BOB) ${ }^{12,13}$ and the Coulomb matrix (CM). ${ }^{13,14}$ Atomic variants of the CM have also been proposed and tested on QM9. ${ }^{15}$ Other representations have also been benchmarked on QM9 (or QM7 which is a smaller but similar data set), such as Fourier series of radial distance distributions, ${ }^{16}$ motifs,${ }^{17}$ the smooth overlap of atomic positions (SOAP) ${ }^{18}$ in combination with regularized entropy match, ${ }^{19}$ constant size descriptors based on connectivity and encoded distance distributions. ${ }^{20}$ Ramakrishnan et al. ${ }^{8}$ introduced a $\Delta$-ML approach, where the difference between properties calculated at coarse/accurate quantum level of theories is being modeled. Furthermore, neural network models, as well as deep tensor neural networks, have recently been proposed and tested on the same or similar data sets. ${ }^{21,22}$ Dral et al. ${ }^{23}$ use such data to machine learn optimal molecule specific parameters for the OM2 ${ }^{24}$ semiempirical method, and orthogonalization tests are benchmarked in Ref. ${ }^{25}$

However, limited work has yet been done in systematically assessing various methods and properties on large sets of the exact same chemicals. ${ }^{26}$ In order to unequivocally establish if ML has the potential to replace hybrid DFT for the screening of properties, one has to demonstrate that ML test errors are systematically lower than estimated hybrid DFT accuracies for all the properties available. This study accomplishes that through a large scale assessment of unprecedented scale: (i) In order to approximate large training set sizes $N$, we included 13 quantum properties from up to $\sim 118 \mathrm{k}$ molecules in training $(90 \%$ of QM9). (ii) We tested multiple regressors (Bayesian ridge regression (BR), linear regression with elastic net regularization $(\mathrm{EN})$, random forest $(\mathrm{RF})$, kernel ridge regression (KRR), neural network (NN) models graph convolutions $(\mathrm{GC})^{27}$ and gated graphs $(\mathrm{GG})^{28}$ ) and (iii) multiple representations including BAML, $\mathrm{BOB}, \mathrm{CM}$, extended connectivity fingerprints (ECFP4), histograms of distance, angle, and dihedral (HDAD), molecular atomic radial angular distribution (MARAD), and molecular graphs (MG). (iv) We investigated all combinations of regressors and representations, except for $\mathrm{MG} / \mathrm{NN}$ which was exclusively used together because GC and GG depend fundamentally on the input representation being a graph instead of a flat feature vector.

The best models for the various properties are: atomization energy at 0 Kelvin (HDAD/KRR), atomization energy at room temperature (HDAD/KRR), enthalpy of atomization at room temperature (HDAD/KRR), atomization of free energy at room temperature (HDAD/KRR), HOMO/LUMO eigenvalue and gap (MG/GC), dipole moment (MG/GC), static polarizability $(\mathrm{MG} / \mathrm{GG})$, zero point vibrational energy (HDAD/KRR), heat capacity at room temperature (HDAD/KRR), and the highest fundamental vibrational frequency (BAML/RF). For training set size of $\sim 118 \mathrm{k}$ 
(90\% of data set) we have found the additional out-of-sample error added by machine learning to be lower or as good as DFT errors at B3LYP level of theory relative to experiment for all properties, and that chemical accuracy (See table 3) is reached, or in sight.

This paper is organized as follows: First we will briefly describe the methods, including data set, model validation protocols, representations, and regressors. In section III, we present the results and discuss them, and section IV concludes the paper.

\section{Method}

\subsection{Data set}

We have used the QM9 data set consisting of $\sim 134 \mathrm{k}$ drug-like organic molecules. ${ }^{9}$ Molecules in the data set consist of $\mathrm{H}, \mathrm{C}, \mathrm{O}, \mathrm{N}$ and $\mathrm{F}$, and contain up to 9 heavy atoms. For each molecule several properties, calculated at DFT level of theory (B3LYP/6-31G(2df,p)), were included. We used: Atomization energy at 0 Kelvin $U_{0}$ $(\mathrm{eV})$; atomization energy at room temperature $U(\mathrm{eV})$; enthalpy of atomization at room temperature $H(\mathrm{eV})$; atomization of free energy at room temperature $G(\mathrm{eV})$; HOMO eigenvalue $\varepsilon_{\text {HOMO }}(\mathrm{eV})$; LUMO eigenvalue $\varepsilon_{\mathrm{LUMO}}$ $(\mathrm{eV})$; HOMO-LUMO gap $\Delta \varepsilon(\mathrm{eV})$; norm of dipole moment $\mu=\sqrt{\sum_{r \in x, y, z}\left(\int d \mathbf{r} n(\mathbf{r}) r\right)^{2}}$ (Debye), where $n(\mathbf{r})$ is the molecular charge density distribution; static isotropic polarizability $\alpha=\frac{1}{3} \sum_{i \in x, y, z} \alpha_{i i}\left(\right.$ Bohr $\left.^{3}\right)$, where $\alpha_{i i}$ is the diagonal element of the polarizability tensor; zero point vibrational energy ZPVE $(\mathrm{eV})$; heat capacity at room temperature $C_{\mathrm{v}}(\mathrm{cal} / \mathrm{mol} / \mathrm{K})$; and the highest fundamental vibrational frequency $\omega_{1}\left(\mathrm{~cm}^{-1}\right)$. For energies of atomization $\left(U_{0}, U, H\right.$ and $G$ ) all models yield very similar errors. We will therefore only discuss $U_{0}$ for the remainder. The 3053 molecules specified in Ref. ${ }^{9}$ which failed SMILES consistency tests were excluded from our study, as well as two linear molecules, leaving $\sim 131 \mathrm{k}$ molecules.

\subsection{Model validation}

Starting from the $\sim 131 \mathrm{k}$ molecules in QM9 after removing the $\sim 3 \mathrm{k}$ molecules (see above) we have created a number of train-validation-test splits. We have splitted the data set into test and non-test sets and varied the percentage of data in test set to explore the effect of amount of data in error rates. Inside the non-test set, we have performed 10 fold cross validation for hyperparameter optimization. That is, for each model $90 \%$ (the training set) of the non-test set is used for training and $10 \%$ (the validation set) is used for hyperparameter selection. For each test/non-test split, we have trained 10 models on different subsets of the non-test set, and we report the mean error on the test set across those 10 models. Note that the non-test set will be referred to as training set in the results section in order to simplify discussion.

In terms of CPU investments necessary for training the respective models we note that $\mathrm{EN} / \mathrm{BR}, \mathrm{RF} / \mathrm{KRR}$, and $\mathrm{GC} / \mathrm{GG}$ required minutes, hours, and multiple days, respectively. Using GPUs could dramatically reduce such timings.

\subsection{DFT errors}

To place the quality of our prediction errors in the right context, experimental accuracy estimates of hybrid DFT become desirable. Here, we summarize literature results comparing DFT at B3LYP level of theory to experiments for the various properties we study. Where data is available, the corresponding deviation from experiment is listed in Table 3, alongside our ML prediction errors (vide infra).

In order to also get an idea of hybrid DFT energy errors for organic molecules, such as the compounds studied herewithin, we refer to a comparison of $\mathrm{PBE}$ and B3LYP results for $6 \mathrm{k}$ constitutional isomers of $\mathrm{C}_{7} \mathrm{H}_{10} \mathrm{O}_{2} \cdot{ }^{8}$ After centering the data by subtracting their mean shift from G4MP2 (177.8 (PBE) and 95.3 (B3LYP) $\mathrm{kcal} / \mathrm{mol}$ ). The remaining MAEs are roughly $\sim 2.5$ and $\sim 3.0 \mathrm{kcal} / \mathrm{mol}$ for B3LYP and PBE, respectively. This is in agreement with what Curtiss et al. ${ }^{29}$ found. They compared DFT 
to experimental values from 69 small organic molecules (of which 47 were substituted with $\mathrm{F}, \mathrm{Cl}$, and S), with up to 6 heavy atoms (not counting hydrogens), and calculated the energies using B3LYP/6-311+G(3df,2p). The resulting mean absolute deviation from experimental values was $2.3 \mathrm{kcal} / \mathrm{mol}$.

Rough hybrid DFT error estimates for dipole moment and polarizability have been obtained from Refs. ${ }^{30}$.The errors are estimated referenced to experimental values, for a data set consisting of 49 molecules with up to 7 heavy atoms $(\mathrm{C}, \mathrm{Cl}, \mathrm{F}, \mathrm{H}, \mathrm{O}, \mathrm{P}$, or $\mathrm{S})$

Frontier molecular orbital energies (HOMO, LUMO and HOMO-LUMO gap) can not be measured directly. However, for the exact (yet unknown) exchange-correlation potential, the Kohn-Sham HOMO eigenvalues correspond to the negative of the vertical ionization potential (IP). ${ }^{31}$ Unfortunately, within hybrid DFT, the precise meaning of the frontier eigenvalues and the gap is less clear, and we therefore refrain from a direct comparison of B3LYP to experimental numbers. Nevertheless, we have included eigenvalues and the gap due to their widespread use for molecular and materials design applications.

Hybrid DFT RMSE estimates with respect to experimental values of ZPVE and $\omega_{1}$ (the highest fundamental vibrational frequency) were published in Ref. ${ }^{32}$ for a set of 41 organic molecules, with up to 6 heavy atoms (not counting hydrogen) and calculated using B3LYP/ccpVTZ.

Normally distributed data has a constant ratio between RMSE and MAE, ${ }^{33}$ which is roughly 0.8. We have used this ratio to approximate the MAE from the RMSE estimates reported for ZPVE and $\omega_{1}$. Deviation of DFT (at the B3LYP/6-311g** level of theory) from experimental heat capacities were reported by DeTar $^{34}$ who obtained errors of 16 organic molecules, with up to 8 heavy atoms (not counting hydrogens).

Note, however, that one should be cautious when referring to these errors: Strictly speaking they can not be compared since different basis sets, molecules, and experiments were used. We also note that all DFT errors in this pa- per are estimated from B3LYP and using other functionals can yield very different errors.

Nevertheless, we feel that the quoted errors provide meaningful guidance as to what one can expect from DFT for each property.

\subsection{Representations}

The design of molecular representations is a long-standing problem in chem-informatics and materials informatics, and many interesting and promising variants have already been proposed. Below, we provide the details on the representations selected for this study. While finalizing our study, competitive alternatives were introduced $^{35,36}$ but have been tested only for energies (and polarizabilities).

\subsubsection{CM and BOB}

The Coulomb matrix $(\mathrm{CM})$ representation ${ }^{14}$ is a square atom by atom matrix, where off diagonal elements are the Coulomb nuclear repulsion terms between atom pairs. The diagonal elements approximate the electronic potential energy of the free atoms. Atom indices in the CM are sorted by the $L^{1}$ norm of each atom's row (or column). The Bag of Bonds (BOB) ${ }^{12}$ representation uses exclusively CM elements, grouping them for different atom pairs into different bags, and sorting them within each bag by their relative magnitude.

\subsubsection{BAML}

The recently introduced BAML (Bonds, angles, machine learning) representation can be viewed as a many-body extension of BOB. ${ }^{11}$ All pairwise nuclear repulsions are replaced by Morse/Lennard-Jones potentials for bonded/non-bonded atoms respectively. Furthermore, three- and four-body interactions between covalently bonded atoms are included using angular and torsional terms, respectively. Parameters and functional forms are based on the universal force field (UFF). ${ }^{37}$ 


\subsubsection{ECFP4}

Extended Connectivity Fingerprints ${ }^{38}$ (ECFP4) are a common representation of molecules in cheminformatics based studies. They are particularly popular for drug discovery. ${ }^{39-41}$ The basic idea, typical also for other cheminformatics descriptors ${ }^{42}$ (e.g. the signature descriptor $^{43,44}$ ) is to represent a molecule as the set of subgraphs up to a fixed diameter (here we use ECFP4, which is a max diameter of 4 bonds). To produce a fixed length vector, the subgraphs can be hashed such that every subgraph sets one bit in the fixed length vector to 1 . In this work, we use a fixed length vector of size 1024 . Note that ECFP4 is based solely on the molecular graph specifying all covalent bonds, e.g. as encoded by SMILES strings.

\subsubsection{MARAD}

Molecular atomic radial angular distribution (MARAD) is an atomic radial distribution function (RDF) based representation. Per atom it consists of three RDFs using Gaussians of interatomic distances, and parallel and orthogonal projections of distances in atom triplets, respectively. Distances between two molecules can be evaluated analytically. Unfortunately, most regressors evaluated in this work, such as $\mathrm{BR}$, EN and RF, do not rely on inner products and distances between representations. We resolve this issue by projecting MARAD onto bins in order to work with all regressors (apart for GG and GC which use MG exclusively). The three body terms in MARAD contain information about both, angles and distances of all atoms involved. This differs from HDA (see below), where distances, and angles are decoupled, and placed in separated bins. Note that unlike BAML or HDAD, there are only two and three-body terms, no four-body terms (dihedral angles) have been included within MARAD.

Details about how the projected MARAD is calculated can be found under in the Supplementary materials.

Further details and characteristics of MARAD will also be discussed in a forthcoming separate in-depth study.

\subsubsection{HD, HDA, and HDAD}

BOB, BAML and MARAD rely on computing functions for given interatomic distances, and/or angles, and/or torsions, and then either project that value on to discrete bins, or sort the values. As a straightforward alternative, we also investigated representations which account directly from pairwise distances, triple-wise angles, and quad-wise dihedral angles through manually generated bins in histograms. The resulting representations in increasing interatomic many-body order are called HD (Histogram of distances), HDA (Histogram of distances and angles), and HDAD (Histogram of distances, angles and dihedral angles). For any given molecule, one iterates through each atom $a_{i}$, producing a set of distances, angle and dihedral angle features for $a_{i}$.

Distance features were produced by measuring the distance between $a_{i}$ and $a_{j}$ (for $i \neq j$ ) for each element pair. The distance features were assigned a label incorporating the atomic symbols of $a_{i}$ and $a_{j}$ sorted alphabetically (with $\mathrm{H}$ last), e.g. if $a_{i}$ was a carbon atom and $a_{j}$ was a nitrogen atom, the distance feature for the atom pair would be labeled C-N. These labels will be used to group all features with the same label into a histogram and allow us to only count each pair of atoms once.

Angle features were produced by taking the principal angles formed by the two vectors spanning from each atom $a_{i}$ to every subset of 2 of its 3 nearest atoms, $a_{j}$ and $a_{k}$. The angle features were labeled by the element type of $a_{i}$, followed by the alphabetically sorted element types (Except for hydrogens, which were listed last) of $a_{j}$ and $a_{k}$. The example where $a_{i}$ is a Carbon atom, $a_{j}$ a Hydrogen atom, $a_{k}$ a Nitrogen would be assigned the label $\mathrm{C}-\mathrm{N}-\mathrm{H}$.

Dihedral angle features were produced by taking the principal angles between two planes. We take $a_{i}$ as the origin, and for each of the four nearest neighbors in turn, labeling the neighbor atom $a_{j}$, and forming a vector $V_{i j}=a_{i} \rightarrow a_{j}$. Then all $\left(\begin{array}{l}3 \\ 2\end{array}\right)$ subsets of the remaining three out of four nearest neighbors of $a_{i}$ are chosen, and labeled as $a_{k}$ and $a_{l}$. This third and fourth atom respectively form two triangular faces when 
paired with $V_{i j}:\left\langle a_{k}, a_{i}, a_{j}\right\rangle$ and $\left\langle a_{l}, a_{i}, a_{j}\right\rangle$. The dihedral angle between the two triangular faces was calculated. These dihedral angle features were labeled with the atomic symbol for $a_{i}$, followed by the atomic symbols for $a_{j}, a_{k}$ and $a_{l}$, sorted alphabetically, with the exception that hydrogens were listed last, e.g. C-C-N-H.

The features from all molecules have been aggregated for each label type to generate a histograms for each label type. Fig. 1 exemplifies this for C-N distances, C-C-C angles, and CC-C-O dihedrals for the entire QM9 data set. Certain typical molecular characteristics can be recognized upon mere inspection. For example, the $\mathrm{CN}$ histogram displays a strong and isolated peak between 1.1 and $1.5 \AA$, corresponding to occurrences of single, double, and triple bonds. For distances above $2 \AA$, peaks at typical radii of second and third covalent bonding shells around $\mathrm{N}$ can be recognized at $2.6 \AA$ and $3.9 \AA$. Also C-C-C angles can be easily interpreted: The peak close to zero and $\pi$ Rad corresponds to geometries where three atoms are part of a linear (alkyne, or nitrile) type of motif. The broad and largest peak corresponds to 120 and 109 degrees, typically observed in $s p^{2}$ and $s p^{3}$ hybridized atoms.

The morphology of each histogram has then been examined to identify apparent peaks and troughs, motivated by the idea that peaks indicate structural commonalities among molecules. Bin centers have been placed at each significant local minimum and maximum (Shown as vertical lines in Fig. 1). Values at 15-25 bin centers have been chosen as a representation for each label type. All bin center values are provided in the Supplementary Material. For each molecule, the collection of features has subsequently been rendered into a fixed-size representation, producing one vector component for each bin center, within each label type. This has been accomplished following a two-step process. (i) Binning and interpolation: Each feature value is projected on the two nearest bins. The relative amount projected on each bin uses linear projection between the two bins. For example: A feature with value 1.7 which lies between two bins placed at 1.0 and 2.0 respectively, contributes 0.3 and 0.7 to the first and second bin respectively. (ii) Reduction: The collection of contributions within each bin of each molecule's feature vector is condensed to a single value by summing all contributions.

\subsubsection{Molecular Graphs}

We have investigated several neural network models which are based on molecular graphs (MG) as representation. The inputs are real-valued vectors associated with each atom and with each pair of atoms. More specifically, we have used the featurization described in Kearnes et al. ${ }^{27}$ with the removal of partial charge and the addition of Euclidean distances to the pair feature vectors. All elements of the feature vector are described in Tables 1 and 2 .

The featurization process was unsuccessful for a small number of molecules (367) because of conversion failures from geometry to rational SMILES string when using OpenBabel ${ }^{45}$ or RDKit, ${ }^{46}$ and were excluded from all results using the molecule graph features.

Table 1: Atom features for the MG representation: Values provided for each atom in the molecule.

\begin{tabular}{l|l}
\hline Feature & Description \\
\hline \hline Atom type & H, C, N, O, F (one-hot). \\
\hline Chirality & R or S (one-hot or null). \\
\hline Formal charge & Integer electronic charge. \\
\hline Ring sizes & $\begin{array}{l}\text { For each ring size (3-8), } \\
\text { the number of rings that } \\
\text { include this atom. }\end{array}$ \\
\hline Hybridization & $\begin{array}{l}s p, s p^{2} \text {, or } s p^{3} \text { (one-hot or } \\
\text { null). }\end{array}$ \\
\hline Hydrogen bonding & $\begin{array}{l}\text { Whether this atom is a hy- } \\
\text { drogen bond donor and/or } \\
\text { acceptor (binary values). }\end{array}$ \\
\hline Aromaticity & $\begin{array}{l}\text { Whether this atom is part } \\
\text { of an aromatic system. }\end{array}$ \\
\hline
\end{tabular}

Note that within a previous draft of this study ${ }^{47}$ we reported biased results for $\mathrm{GC} / \mathrm{GG}$ models due to use of Mulliken partial charges within the MG representation. All MG results presented herewithin have been obtained without any Mulliken charges in the representation. Model hyper parameters for the GC 
Table 2: Atom pair features for the MG representation: Values provided for each pair of atoms in the molecule.

\begin{tabular}{l|l}
\hline Feature & Description \\
\hline \hline Bond type & $\begin{array}{l}\text { Single, double, triple, or aro- } \\
\text { matic (one-hot or null). }\end{array}$ \\
\hline Graph distance & $\begin{array}{l}\text { For each distance (1-7), } \\
\text { whether the shortest path } \\
\text { between the atoms in the } \\
\text { pair is less than or equal to } \\
\text { that number of bonds (binary } \\
\text { values). }\end{array}$ \\
\hline Same ring & $\begin{array}{l}\text { Whether the atoms in the pair } \\
\text { are in the same ring. }\end{array}$ \\
\hline Spatial distance & $\begin{array}{l}\text { The euclidean distance be- } \\
\text { tween the two atoms. }\end{array}$ \\
\hline
\end{tabular}

model, however, still correspond to the previously obtained hyper parameter search.

\subsection{Regressors}

For all methods, we first standardized the property values so that all properties have zero mean and unit standard deviation.

\subsubsection{Kernel Ridge Regression}

$\mathrm{KRR}^{48-51}$ is a type of regression with regularization $^{52}$ which uses kernel functions as basis set. A property $p$ of a query molecule $\mathbf{m}$ is predicted by a sum of weighted kernel functions $K\left(\mathbf{m}, \mathbf{m}_{i}^{\text {train }}\right)$ between $\mathbf{m}$ and all molecules $\mathbf{m}_{i}^{\text {train }}$ in the training set,

$$
p(\mathbf{m})=\sum_{i}^{N} \alpha_{i} K\left(\mathbf{m}, \mathbf{m}_{i}^{\text {train }}\right)
$$

where $\alpha_{i}$ are regression coefficients, obtained by minimizing the Euclidean distance between the estimated and the reference property of all molecules in the training set. We used Laplacian and Gaussian kernels as implemented by scikit-learn ${ }^{53}$ for all representations.

The level of noise in our data is very low so strong regularization is not necessary. We have set the regularization parameter to $10^{-9}$, and we note that prediction errors change negligibly when altering it to $10^{-10}$. Kernel widths were chosen by screening values on a base- 2 
logarithmic grid for the 10 percent training set (from 0.25 to 8192 for Gaussian kernel and 0.1 to 16384 for Laplacian kernel). In order to simplify the width screening, prior to learning all feature vectors were normalized (scaling the input vector by the mean norm across the training set) by the Euclidean norm for the Gaussian kernel and the Manhattan norm for the Laplacian kernel.

\subsubsection{Bayesian Ridge Regression}

We use $\mathrm{BR}^{54}$ as is implemented in scikitlearn. ${ }^{53} \mathrm{BR}$ is a linear model with a $L^{2}$ penalty on the coefficients. Unlike Ridge Regression where the strength of that penalty is a regularization hyperparameter which must be set, in Bayesian Ridge Regression the optimal regularizer is estimated from the data.

\subsubsection{Elastic Net}

Also $\mathrm{EN}^{55}$ is a linear model. Unlike BR, the penalty on the weights is a mix of $L^{1}$ and $L^{2}$ terms. In addition to the regularization hyperparameter for the weight penalty, Elastic net has an additional hyperparameter 11 ratio to control the relative strength of the $L^{\overline{1}}$ and $L^{2}$ weight penalties. We used the scikit-learn ${ }^{53}$ implementation and set 11 ratio $=0.5$. We then did a hyperparameter search on regularizing parameter in a base 10 logarithmic grid from $1 e-6$ to 1.0 .

\subsubsection{Random Forest}

We use $\mathrm{RF}^{56}$ as implemented in scikit-learn. ${ }^{53}$ $\mathrm{RF}$ regressors produce a value by averaging many individual decision trees fitted on randomly resampled sets of the training data. Each node in each decision tree is a threshold of one input feature. Early experiments did not reveal strong differences in performance based on the number of trees used, once a minimal number was reached. We have used 120 trees for all regressions.

\subsubsection{Graph Convolutions}

We have used the GC model as described in Kearnes et al. ${ }^{27}$, with several structural modifications and optimized hyperparameters. The graph convolution model is built on the concepts of "atom" layers (one real vector associated with each atom) and "pair" layers (one real vector associated with each pair of atoms). The graph convolution architecture defines operations to transform atom and pair layers to new atom and pair layers. There are three structural changes to the model used herewithin when compared to the one described in Kearnes et al. ${ }^{27}$. We describe these briefly here with details in the Supplementary Material. First, we have removed the "Pair order invariance" property by simplifying the $(A \rightarrow P)$ transformation. Since the model only uses the atom layer for the molecule level features, pair order invariance is not needed. Second, we have used the Euclidean distance between atoms. In the $(P \rightarrow A)$ transformation, we divide the value from the convolution step by a series of distance exponentials. If the original convolution for an atom pair $(a, b)$ with distance $d$ produces a vector $V$, we concatenate the vectors $V$, $\frac{V}{d^{1}}, \frac{V}{d^{2}}, \frac{V}{d^{3}}$, and $\frac{V}{d^{6}}$ to produce the transformed value for the pair $(a, b)$. Third, we have followed other work on neural networks based on chemical graphs ${ }^{57}$ which uses a sum of softmax operations to convert a real valued vector to a sparse vector and sum those sparse vectors across all the atoms. We use the same operation here along with a simple sum across the atoms to produce molecule level features from the top atom layer. We have found that this works as well or better than the Gaussian histograms first used in GC. ${ }^{27}$ To optimize the network, we have searched the hyperparameter space using Gaussian Process Bandit Optimization $^{58}$ as implemented by HyperTune. ${ }^{59}$ The hyperparameter search has been based on the evaluation of the validation set for a single fold of the data. Further details including parameters, and search ranges chosen for this paper are listed in the Supplementary materials. 


\subsubsection{Gated Graph Neural Networks}

We have used the GG Neural Networks model (GG) as described in Li et al. ${ }^{28}$. Similar to the GC model, it is a deep neural network whose input is a set of node features $\left\{x_{v}, v \in G\right\}$, and an adjacency matrix $A$ with entries in a discrete set $S=\{0,1, \cdots, k\}$ to indicate different edge types. It has internal hidden representations for each node in the graph $h_{v}^{t}$ of dimension $d$ which it updates for $T$ steps of computation. Its output is invariant to all graph isomorphisms, meaning the order of the nodes presented to the model does not matter. To include the most relevant distance information we distinguish four different covalent bonding types (single, double, triple, aromatic). For all remaining atom-pairs we bin them by their interatomic distance [in $\AA]$ into 10 bins: [0, 2], [2,2.5], [2.5,3], [3,3.5], $[3.5,4],[4,4.5],[4.5,5],[5,5.5],[5.5,6]$, and $[6, \infty]$. Using these bins, the adjacency matrix has entries in an alphabet of size $14(\mathrm{k}=14)$, indicating bond type for covalently bonded atoms, and distance bin for all other atoms. We have trained the GG model on each target property individually. Further technical details are specified in the Supplementary materials.

\section{Results and discussion}

\subsection{Overview}

We present an overview of the most relevant numerical results in Table 3. It contains the test errors for all combinations of regressors and representations and properties for models trained on $\sim 118 \mathrm{k}$ molecules. The best models for the respective properties are $U_{0}(\mathrm{HDAD} / \mathrm{KRR})$, $\varepsilon_{\text {HOMO }}(\mathrm{MG} / \mathrm{GC}), \quad \varepsilon_{\text {LUMO }}(\mathrm{MG} / \mathrm{GC}), \quad \Delta \varepsilon$ (MG/GC), $\mu(\mathrm{MG} / \mathrm{GC}), \alpha(\mathrm{MG} / \mathrm{GG}), \mathrm{ZPVE}$ $(\mathrm{HDAD} / \mathrm{KRR}), \quad C_{\mathrm{v}}(\mathrm{HDAD} / \mathrm{KRR})$, and $\omega_{1}$ $(\mathrm{BAML} / \mathrm{RF})$. We do not show results for the other three energies, $U(T=298 K), H(T=$ $298 K), G(T=298 K)$ since identical observations as for $U_{0}$ can be made.

Overall, NN and KRR regressors perform well for most properties. The ML out-of-sample errors outperform DFT accuracy at B3LYP level of theory and reach chemical (target) accuracy, both defined alongside in table 3, for $U_{0}(\mathrm{HDAD} / \mathrm{KRR}$ and $\mathrm{MG} / \mathrm{GG}), \mu(\mathrm{GC})$, $C_{\mathrm{v}}(\mathrm{HDAD} / \mathrm{KRR})$, and $\omega_{1}(\mathrm{BAML} / \mathrm{KRR}$, $\mathrm{MG} / \mathrm{GC}, \mathrm{HDAD} / \mathrm{KRR}, \mathrm{BOB} / \mathrm{KRR}, \mathrm{HD} / \mathrm{KRR}$ and $\mathrm{MG} / \mathrm{GG}$ ). For the remaining properties ( $\varepsilon_{\text {HOMO }}, \varepsilon_{\text {LUMO }}, \Delta \epsilon, \alpha$, and ZPVE) the best models come within a factor 2 of target accuracy, while all (except $\varepsilon_{\text {HOMO }}, \varepsilon_{\text {LUMO }}$ and $\Delta \epsilon$ ) where we don't have reliable data. outperforming DFT accuracy.

In Fig. 2 out-of-sample errors as a function of training set size (learning curves) are shown for all properties and representations with the best corresponding regressor. It is important to note that all models on display systematically improve with training set size, exhibiting the typical linearly decaying behavior on a log-log plot. ${ }^{11,61}$ Errors for most models shown decay with roughly the same slopes, indicating similar exponents in the power-law of error decay. Notable exceptions, i.e. property models with considerably steeper learning curves (Slopes and off-sets of all learning curves can be found in Tables S4 and S5 in the Supplementary Material ), are $\mathrm{MG} / \mathrm{GC}$ for $\mu, \mathrm{MG} / \mathrm{GG}$ and $\mathrm{HDAD} / \mathrm{KRR}$ for $\alpha, \mathrm{CM} / \mathrm{KRR}$ and $\mathrm{BOB} / \mathrm{KRR}$ for $\left\langle\mathrm{R}^{2}\right\rangle, \mathrm{HDAD} / \mathrm{KRR}$ and $\mathrm{MG} / \mathrm{GG}$ for $U_{0}$, and $\mathrm{MG} / \mathrm{GG}$ for $\omega_{1}$. These results suggest that the specified representations capture particularly well the effective dimensionality of the corresponding property in chemical space.

\subsection{Regressors}

Inspection of Table 3 indicates that the regressors can roughly be ordered by performance, independent of property and representation: $\mathrm{GC}>\mathrm{GG}>\mathrm{KRR}>\mathrm{RF}>\mathrm{BR}>\mathrm{EN}$. It is noteworthy how EN, BR, and RF regressors perform substantially worse than GC/GG/KRR. The bad performance of EN and BR is due to their low model capacities. This can also be seen from the learning curves of all regressors presented in Figures S1 to S6 of the Supplementary Material. The performance of BR and EN improves only slightly with increased training set size and even gets worse for some property/representation combinations. These two regressors also exhibit very similar learn- 
Table 3: MAE on out-of-sample data of all representations for all regressors and properties at $\sim 118 \mathrm{k}$ (90\%) training set size. Regressors include linear regression with elastic net regularization (EN), Bayesian ridge regression (BR), random forest (RF), kernel ridge regression (KRR) and molecular graphs based neural networks $(\mathrm{GG} / \mathrm{GC})$. The best combination for each property are highlighted in bold. Additionally, the table contains mean MAE of representations for each property and regressor; and normalized, by MAD (See Table 4), mean MAE (NMMAE) over all properties for each regressor/representation combination.

\begin{tabular}{|c|c|c|c|c|c|c|c|c|c|c|c|}
\hline & & $U_{0} \quad \varepsilon_{\mathrm{H}}$ & HOMO $\varepsilon$ & LUMO & $\Delta \varepsilon$ & $\mu$ & $\alpha$ & ZPVE & $C_{\mathrm{v}}$ & $\omega_{1}$ & NMMAE \\
\hline & & $\mathrm{eV}$ & $\mathrm{eV}$ & $\mathrm{eV}$ & $\mathrm{eV}$ & Debye & Bohr $^{3}$ & $\mathrm{eV}$ & $/ \mathrm{molK}$ & $\mathrm{cm}^{-1}$ & arb. u. \\
\hline \multirow{8}{*}{ EN } & $\bar{M}$ & 0.911 & 0.338 & 0.631 & 0.722 & $\overline{0.844}$ & 1.33 & 0.0265 & 0.906 & 131.0 & 0.423 \\
\hline & B & 602 & 0.283 & 0.521 & 0.614 & 0.763 & 1.2 & 0232 & 0.7 & 81 & 35 \\
\hline & BAML & 0.212 & 0.186 & 0.275 & 0.339 & 0.686 & 0.793 & .0129 & 0.439 & 60.4 & .231 \\
\hline & ECFP4 & 3.68 & 0.224 & 0.344 & 0.383 & 0.737 & 3.45 & 0.27 & 1.51 & 86.6 & .462 \\
\hline & HDAD & 0.0983 & 30.139 & 0.238 & 0.278 & 0.563 & 0.437 & 0.00647 & 0.0876 & 94.2 & .183 \\
\hline & & 0.192 & 0.203 & 0.299 & 0.36 & 0.705 & 0.638 & 0.00949 & 0.195 & 104.0 & .236 \\
\hline & MAR & 0.183 & 0.222 & 0.305 & 0.391 & 0.707 & 0.698 & 0.00808 & 0.206 & 108.0 & .256 \\
\hline & Mean & 0.84 & 0.228 & 0.373 & 0.441 & 0.715 & 1.22 & 0.0509 & 0.578 & 95.1 & \\
\hline \multirow{8}{*}{$\mathrm{R}$} & $\mathrm{CM}$ & 0.911 & 0.338 & $\begin{array}{l}0.632 \\
\end{array}$ & $\begin{array}{l}0.723 \\
\end{array}$ & 0.844 & 1.33 & 0.0265 & 0.907 & $\begin{array}{l}131.0 \\
\end{array}$ & .424 \\
\hline & $\mathrm{BOB}$ & 0.586 & 0.279 & 0.521 & 0.614 & 0.761 & 1.1 & 0.0222 & 0.684 & 80.9 & .343 \\
\hline & BAML & 0.202 & 0.183 & 0.275 & 0.339 & 0.685 & 0.785 & 0.0129 & 0.444 & 60.4 & .229 \\
\hline & ECFP4 & 3.69 & 0.224 & 0.344 & 0.383 & 0.737 & 3.45 & 0.27 & 1.51 & 86.7 & 0.462 \\
\hline & HDAD & 0.0614 & 40.14 & 0.238 & 0.278 & 0.565 & 0.4 & 0.00318 & 0.0787 & 94.8 & 0.182 \\
\hline & HD & 0.171 & 0.203 & 0.298 & 0.359 & 0.705 & 0.633 & 0.00693 & 0.19 & 104.0 & 0.235 \\
\hline & MARAL & 0.171 & 0.184 & 0.257 & 0.315 & 0.647 & 0.533 & 0.00854 & 0.201 & 103.0 & 0.226 \\
\hline & Mean & 0.828 & 0.221 & 0.367 & 0.43 & 0.706 & 1.19 & 0.05 & 0.574 & 94.5 & \\
\hline \multirow{8}{*}{$\mathrm{RF}$} & $\mathrm{CM}$ & 0.431 & 0.208 & 0.302 & 0.373 & .608 & $\overline{110}$ & 0.0199 & 0.777 & 13.2 & .239 \\
\hline & $\mathrm{BOB}$ & 0.202 & 0.12 & 0.137 & 0.164 & 0.45 & & 11 & 0.443 & 3.55 & .142 \\
\hline & BAML & 0.2 & 0.107 & 0.118 & 0.141 & 0.434 & 0.638 & .0132 & 0.451 & 2.71 & 0.141 \\
\hline & ECFP4 & 3.66 & 0.143 & 0.145 & 0.166 & 0.483 & 3.7 & 0.242 & 1.57 & 14.7 & 0.349 \\
\hline & HDAD & 1.44 & 0.116 & 0.136 & 0.156 & 0.454 & 1. & 0.0525 & 0.895 & 3.45 & 0.198 \\
\hline & HD & 1.39 & 0.126 & 0.139 & 0.15 & 0.457 & 1.6 & 0.0497 & 0.879 & 4.18 & 0.197 \\
\hline & MARA & 0.21 & 0.178 & 0.243 & 0.311 & 0.607 & 0.676 & 0.0102 & 0.311 & 19.4 & 0.199 \\
\hline & Mean & 1.08 & 0.142 & 0.174 & 0.209 & 0.499 & 1.43 & 0.0569 & 0.761 & 8.74 & \\
\hline \multirow{8}{*}{ KRR } & $\mathrm{CN}$ & 0.128 & 0.133 & 3 & 0.229 & 0 & 0.4 & 48 & 0.118 & 33 & .136 \\
\hline & & 0.0667 & 70.0948 & 0 . & 0.148 & 0 . & & 0.00364 & 0.0917 & & 098 \\
\hline & ML & 0.0519 & 90.0946 & $\begin{array}{ll}60.121 \\
\end{array}$ & 0.152 & 0.4 & & 0.00331 & 0.082 & 19.9 & 105 \\
\hline & ECFP4 & 4.25 & 0.124 & 0.133 & 0.174 & 0. & 4. & 0.248 & 1.84 & 26.7 & 0.383 \\
\hline & HDAD & 0.025 & 10.0662 & 0.0842 & 0.107 & & & 0.00191 & 0.0441 & 23 & 0.076 \\
\hline & $\mathrm{H}$ & 0.0644 & 40.0874 & 40.113 & 0.143 & 0.364 & 0.2 & 0.00316 & 0.0844 & +21 & 0.09 \\
\hline & MAR & 0.0529 & 90.103 & 0.124 & 0.163 & 0.468 & & 0.00301 & 0.0758 & 21.3 & 0.112 \\
\hline & Mean & 0.662 & 0.101 & 0.126 & 0.159 & 0.427 & 0.859 & 0.0383 & 0.333 & 22.7 & \\
\hline GG & $\mathrm{N}$ & 0.0421 & 10.0567 & 0.0628 & 0.0877 & 70.247 & 0.1 & 0.00431 & 0.0837 & 6.22 & $\overline{0.060}$ \\
\hline $\mathrm{Cr}$ & MG & 0.15 & 0.0549 & 90.062 & 0.0869 & $\begin{array}{lll}9 & 0.101\end{array}$ & 0.232 & 0.00966 & 0.097 & 4.76 & .04 \\
\hline
\end{tabular}

ing curves and BR performs only slightly better than EN for most combinations. The only clear exception to this rule is for ZPVE and $U_{0}$ together with HDAD, where BR performs significantly better than EN. Also, BR and EN errors rapidly converge to a constant w.r.t. training set size for all representations and properties, except for HDAD, which is the only representa- tion which has a noteworthy improvement with increased training set size for some properties. The constant learning rates are not surprising as (a) the number of free regression parameters in BR and EN is relatively small and does not grow with training set size, and as (b) the underlying model is a linear combination with small flexibility. This behavior implies error 
Table 4: Mean and mean absolute deviation (MAD) for all properties in the QM9 data set, as well as target MAE, and DFT (at B3LYP level of theory) MAE relative to experiment for each property, and the number of molecules used to estimate the values (In parentheses of DFT row). The target accuracies taken from Ref. ${ }^{13}$ Target accuracy for energies of atomization, and orbital energies were set to $1 \mathrm{kcal} / \mathrm{mol}$, which is generally accepted as (or close to) chemical accuracy within the chemistry community. Target accuracies used for $\mu$ and $\alpha$ are $0.1 \mathrm{D}$ and $0.1 \mathrm{Bohr}^{3}$ respectively, which is within the error of CCSD relative to experiments. ${ }^{30}$ Target accuracies used for $\omega_{1}$ and ZPVE are $10 \mathrm{~cm}^{-1}$, which is slightly larger than $\operatorname{CCSD}(\mathrm{T})$ error for predicting frequencies. ${ }^{60}$ Target accuracies used for $C_{\mathrm{v}}$ were not explained in article. ${ }^{13}$ Section 2.3 discusses how the errors for DFT where obtained.

\begin{tabular}{|c|c|c|c|c|c|c|c|c|c|}
\hline & $\overline{U_{0}}$ & $\varepsilon_{\mathrm{HOMO}}$ & $\varepsilon_{\mathrm{LUMO}}$ & $\Delta \varepsilon$ & $\mu$ & $\alpha$ & ZPVE & $\bar{C} C_{\mathrm{v}}$ & $\omega_{1}$ \\
\hline & $\mathrm{eV}$ & $\mathrm{eV}$ & $\mathrm{eV}$ & $\mathrm{eV}$ & Debye & Bohr $^{3}$ & $\mathrm{eV}$ & $\mathrm{al} / \mathrm{molK}$ & $\mathrm{cm}^{-1}$ \\
\hline Mean & -76.6 & -6.54 & 0.322 & 6.86 & 2.67 & 75.3 & 4.06 & 31.6 & 3500 \\
\hline MAD & 8.19 & 0.439 & 1.05 & 1.07 & 1.17 & 6.29 & 0.717 & 3.21 & 238 \\
\hline Target & 0.043 & 0.043 & 0.043 & 0.043 & 0.10 & 0.10 & 0.0012 & 0.050 & 10 \\
\hline DFT & $0.10(69)$ & NA & $\mathrm{NA}$ & NA & $0.10(49)$ & $0.4(49)$ & $0.0097(41)$ & $0.34(16)$ & $28(4$ \\
\hline
\end{tabular}

convergence already for relatively small training sets.

RF performs poorly compared to GC, GG and KRR for all properties except for $\omega_{1}$, the highest lying fundamental vibrational frequency in each molecule. For this property RF yields an astounding performance with out-of-sample errors as small as single digit $\mathrm{cm}^{-1}$. B3LYP achieves a mean absolute error of only $28 \mathrm{~cm}^{-1}$ with respect to experiment. ${ }^{32}$ The distribution of $\omega_{1}$, Fig. 1 of reference, ${ }^{13}$ suggests a simple reason for this: There are three distinct peaks which correspond to typical C-H, N-H and O-H stretch vibrations in increasing order. Therefore the principal learning task in this property is to detect if there is an $\mathrm{OH}$ group, and if not if there is an $\mathrm{NH}$ group. If neither group is present, $\mathrm{CH}$ will yield the vibration with the highest frequency. As such, this is essentially about classifying which bonds are present in the molecule. RF works by fitting a decision tree to the target property. Each branch in the tree is based on an inequality of one entry in the representation. RF should therefore be able to identify which bonds are present in a molecule, simply by looking at the entries in the each element pair, and/or triplet bin of the representations. For RF, a fractional importance can be assigned to each input feature (the sum of all importances is 1.0). Analyzing the importance of the bins in HDAD of the RF model reveals that the three bins with highest impor- tance are: $\mathrm{O}-\mathrm{H}$ placed at $0.961 \AA, \mathrm{N}-\mathrm{H}$ placed at at $1.01 \AA$ and $\mathrm{C}-\mathrm{C}-\mathrm{H}$ at 3.138 radians with an importance of 0.587, 0.305 and 0.064 respectively. These three first bins constitute $\sim 96 \%$ of the prediction of $\omega_{1}$ and distances of the $\mathrm{O}-\mathrm{H}$ and N-H bins are very similar to $\mathrm{O}-\mathrm{H}$ and $\mathrm{N}-\mathrm{H}$ bond lengths. C-C-H is placed on $\sim \pi$ radians which means that it has to correspond to a linear C-C-H (alkyne) chain which implies that the two carbons must be bonded by a triple bond (typically the $\mathrm{C}-\mathrm{H}$ with the lowest $\mathrm{pK}_{a}$ and the highest $\mathrm{C}-\mathrm{H}$ stretch vibration).

KRR performs remarkably well on average. For extensive energetic properties it yields the lowest overall errors in combination with HDAD and BOB, respectively. Its outstanding performance is not unsurprising in light of the multiple previous ML studies dealing with compositional as well as configurational spaces. The neural network flavors GC and GG, however, yield better performance on average, and the lowest errors for all electronic (mostly intensive) properties, i.e. dipole moment, polarizability, HOMO/LUMO eigenvalues and gaps. A possible explanation for this property dependent difference in performance between KRR and NN could be the inherent respective additive and multiplicative nature of these regressors. The energy being extensive, it is consistent with this picture that effective, quasiparticle based linear KRR based estimates have recently been reported to deliver very accurate 

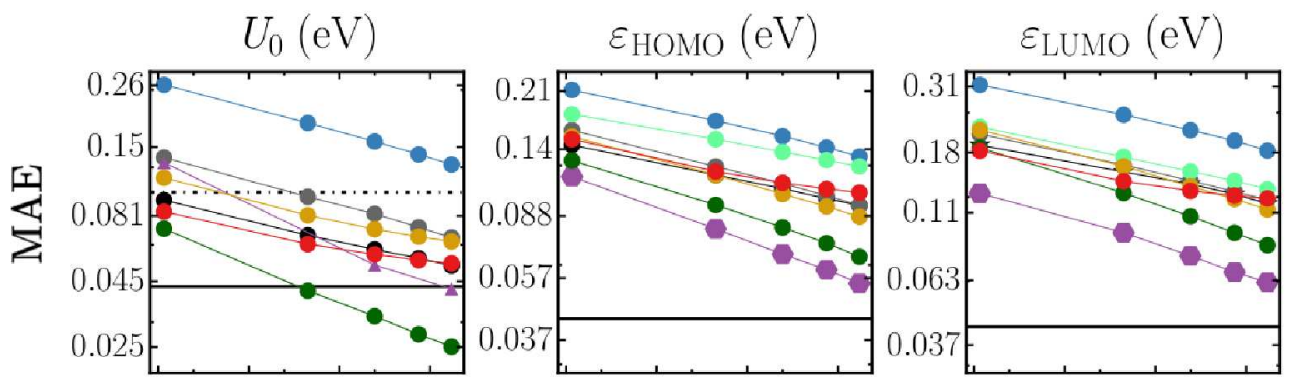

Representations
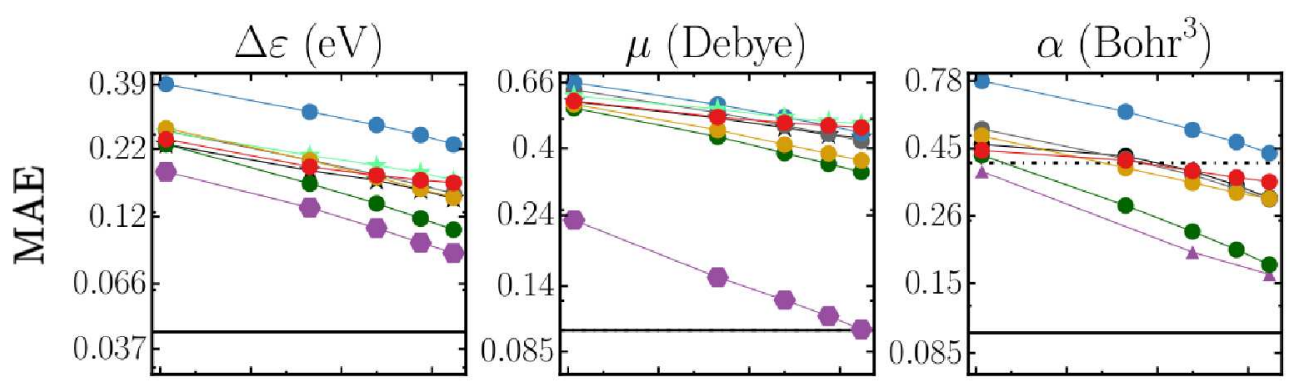

- BAML

- BOB

- $\mathrm{CM}$

ECCFP4

- MG

HDAD

- HD

- MARAD
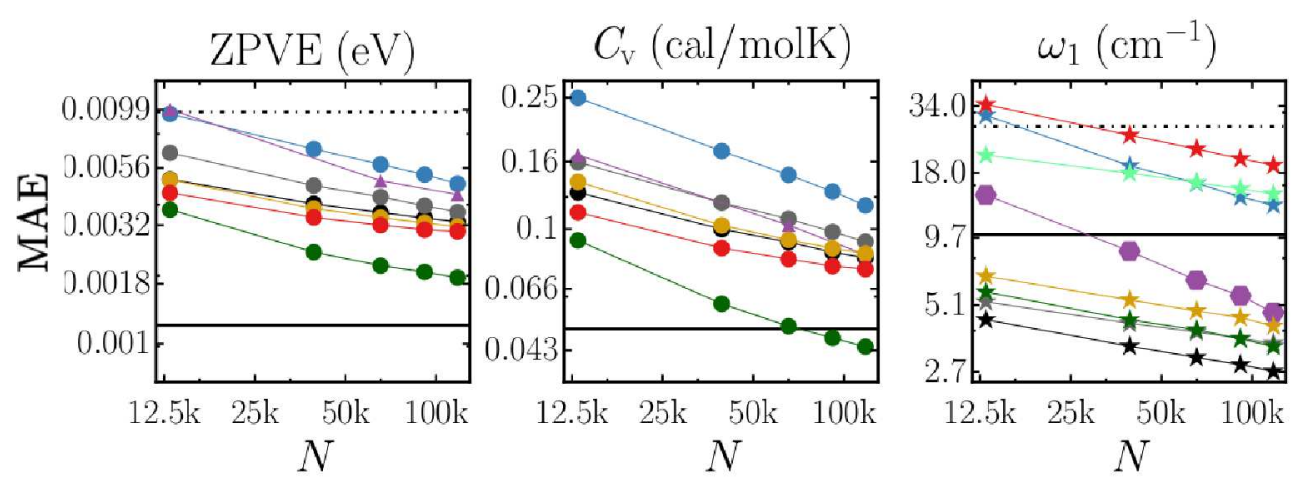

Figure 2: Learning curves (mean absolute error (MAE) as a function of training set size $N$ ) for 10 properties of QM9 molecules, displaying the best regressor for each representation and property. Horizontal solid lines correspond to target accuracies, vertical dotted lines correspond to approximated B3LYP accuracies (unless off-chart), see also table 3. Note that due to its poor performance ECFP4 results have been excluded for $\alpha, \mathrm{ZPVE}, \mathrm{U}$ and $\mathrm{C}_{\mathrm{v}}$.

predictions which can scale. ${ }^{62}$

\subsection{Representations}

As one would expect, HDAD contains more relevant information and thus it always out- performs HD when using KRR. Tests also showed that an HDA representation systematically yields errors in between HDAD and HD, and similar observations hold for BR and EN regressor. In the case of $\mathrm{RF}$, however, we observe little difference between HDAD and HD, 
and HD can even yield slightly lower errors than HDAD. In our opinion, this is due to the additional bins of angles and dihedrals rather adding noise than signal. By contrast, the separation of distances, angles and dihedral angles into different bins is not a problem for the KRR methods because the kernels used are purely distance based. This makes it possible for KRR to exploit the extra three- and four-body information in HDAD and to gain an advantage over HD. We note however that the remarkable performance of HDAD is possible despite its striking simplicity. As illustrated in Fig. 1 and discussed above, characteristic chemical behavior can be directly obtained by human inspection of HDAD. As such, HDAD corresponds to a representation very much "Occam's razor style". Unfortunately, due to its discrete nature and its origin in sorting distances, HDAD will suffer from lack of differentiability, which might limit its applicability when modeling forces or other non-equilibrium properties.

MARAD, containing similar information as HDA, performs similarly to BAML-yet, MARAD requires no prior knowledge about the physics encoded in the universal force-field such as electronic hybridization states, bondorder, or functional potential shapes (Morse, Lennard-Jones, harmonic angular potentials, or sinusoidal dihedrals). BOB and CM, previously state of the art, result in relatively poor performance. ECFP4 produces out-of-sample errors on par or slightly better than $\mathrm{CM} / \mathrm{KRR}$ for intensive properties ( $\mu$, HOMO/LUMO eigenvalues and gap), however the model produces errors that are off-the-chart for all extensive properties $\left(\alpha, \mathrm{ZPVE}, U_{0}\right.$ and $\left.C_{\mathrm{V}}\right)$.

\section{Conclusions}

We have benchmarked many combinations of regressors and representations on the same QM9 data set consisting of $\sim 131 \mathrm{k}$ organic molecules. For all properties, the best ML model prediction errors reach the accuracy of DFT at B3LYP level with respect to experiment. For 7 out of 12 distinct properties (atomization energies, heat-capacity, $\omega_{1}, \mu$ ) out- of-sample errors reach levels on par with chemical accuracy, or better, using a training set size of $\sim 118 \mathrm{k}$ (90\% of QM9 data set) molecules. For the remaining properties $\alpha, \varepsilon_{\mathrm{HOMO}}, \varepsilon_{\mathrm{LUMO}}$, $\Delta \epsilon$, and ZPVE, errors of the best models come within a factor 2 of chemical accuracy.

Regressors EN, BR, and RF lead to rather high out-of-sample errors, while KRR and graph based NN regressors compete for the lowest errors. We have found that GC, GG, and KRR have best performance across all properties, except for the highest vibrational frequency for which RF performs best. There is no single representation and regressor combination that works best for all properties (though forthcoming work with further improvements to the GG based models indicates best in class performance across all properties $\left.{ }^{63}\right)$. For intensive electronic properties ( $\mu, \mathrm{HOMO} / \mathrm{LUMO}$ eigenvalues and gap) we have found $\mathrm{MG} / \mathrm{NN}$ to yield the highest predictive power, while HDAD/KRR corresponds to the most accurate model for extensive properties $\left(\alpha, \mathrm{ZPVE}, U_{0}\right.$ and $C_{\mathrm{V}}$ ). The latter point is remarkable when considering the simplicity of KRR, being just a linear expansion of property in training set, and HDAD, being just histograms of distances, angles, dihedrals. Using BR and EN is not recommended if accuracy is of importance, both regressors perform worse across all properties. Apart from predicting highest fundamental vibrational frequency best, RF based models deliver rather unsatisfactory performance. The ECPF4 based models have shown poor general performance in comparison to all other representations studied; it is not recommended for investigations of molecular properties.

We should caution the reader that all our results refer to equilibrium structures of a set of only $\sim 131 \mathrm{k}$ organic molecules. While $\sim 131 \mathrm{k}$ molecules might seem sufficiently large to be representative, this number is dwarfed in comparison to chemical space, i.e. the space populated by all theoretically stable molecules, estimated to exceed $10^{60}$ for medium sized organic molecules. ${ }^{64}$ Furthermore, ML models for predicting properties of molecules in nonequilibrium or strained configurations might require substantially more training data. This

\section{ACS Paragon Plus Environment}


point is also of relevance because some of the highly accurate models described herewithin (MG based) currently use bond based graph connectivity in addition to distance, raising questions about the applicability to reactive processes.

In summary, for the organic molecules studied, we have collected numerical evidence which suggests that the out-of-sample error of ML is consistently better than estimated DFT at B3LYP level accuracy. While this is no guarantee that ML models would reach same error levels if more accurate, explicitly electron correlated or experimental reference data was used, previous studies indicate that similar performance can be expected when using higher levels of theory. ${ }^{8}$ More specifically, one might intuitively expect that going beyond hybrid DFT to higher quality data (either wavefunction basedQM or experiment) in terms of reference methods would represent a more challenging learning problem, and therefore imply the need for larger training set sizes. Results in Ref., ${ }^{8}$ however, suggest that ML models can predict the differences between HF and MP2, CCSD, and $\operatorname{CCSD}(\mathrm{T})$ equally well using the same training set.

As such, we conclude that future reference datasets for training state-of-the-art machine learning models of molecular properties should preferably use reference levels of theory which go beyond DFT at B3LYP level of accuracy. While it seems unlikely that for each class of molecules, hundreds of thousands of experimental training data points will become available in the foreseeable future, it might well be possible to reach such scale using efficient implementations of explicit electron correlated methods within high-performance computing campaigns. Finally, we note that future work could deal with improving representations and regressors, with the goal of reaching similar predictive power using less data.

Acknowledgement The authors thank Dirk Bakowies for helpful comments, and Adrian Roitberg for pointing out an issue with the use of partial charges in the neural net models in an earlier version of this paper. O.A.v.L. ac- knowledges support from the Swiss National Science foundation (No. PP00P2_138932, 310030 160067), the research fund of the University of Basel, and from Google. This material is based upon work supported by the Air Force Office of Scientific Research, Air Force Material Command, USAF under Award No. FA9550-15-1-0026. This research was partly supported by the NCCR MARVEL, funded by the Swiss National Science Foundation. Some calculations were performed at sciCORE (http://scicore.unibas.ch/) scientific computing core facility at University of Basel.

\section{$5 \mathrm{SI}$}

Supplementary information regarding raw data, MARAD representation, graph convolutions, gated graphs, random forests, and learning curves are reported, as well as root mean square errors for ML predictions after training on the largest training set. 


\section{References}

(1) Hohenberg, P.; Kohn, W. Inhomogeneous Electron Gas. Phys. Rev. 1964, 136, B864.

(2) Kohn, W.; Sham, L. J. Self-Consistent Equations Including Exchange and Correlation Effects. Phys. Rev. 1965, 140, A1133.

(3) Burke, K. Perspective on density functional theory. J. Chem. Phys. 2012, 136, 150901 .

(4) Koch, W.; Holthausen, M. C. A Chemist's Guide to Density Functional Theory; Wiley-VCH, 2002.

(5) Cohen, A. J.; Mori-Sánchez, P.; Yang, W. Challenges for Density Functional Theory. Chem. Rev. 2012, 112, 289-320.

(6) Plata, R. E.; Singleton, D. A. A Case Study of the Mechanism of AlcoholMediated Morita Baylis-Hillman Reactions. The Importance of Experimental Observations. J. Am. Chem. Soc. 2015, 13\%, 3811-3826.

(7) Medvedev, M. G.; Bushmarinov, I. S.; Sun, J.; Perdew, J. P.; Lyssenko, K. A. Density functional theory is straying from the path toward the exact functional. Science 2017, 355, 49-52.

(8) Ramakrishnan, R.; Dral, P. O.; Rupp, M.; von Lilienfeld, O. A. Big Data Meets Quantum Chemistry Approximations: The $\Delta$-Machine Learning Approach. J. Chem. Theory Comput. 2015, 11, 2087-2096.

(9) Ramakrishnan, R.; Dral, P. O.; Rupp, M.; von Lilienfeld, O. A. Quantum chemistry structures and properties of 134 kilo molecules. Sci. Data 2014, 1, 140022.

(10) Ruddigkeit, L.; van Deursen, R.; Blum, L. C.; Reymond, J.-L. Enumeration of 166 Billion Organic Small Molecules in the Chemical Universe Database GDB-17. J. Chem. Inf. Model. 2012, 52, 2864-2875.
(11) Huang, B.; von Lilienfeld, O. A. Communication: Understanding molecular representations in machine learning: The role of uniqueness and target similarity. J. Chem. Phys 2016, 145, 161102.

(12) Hansen, K.; Biegler, F.; Ramakrishnan, R.; Pronobis, W.; von Lilienfeld, O. A.; Müller, K.-R.; Tkatchenko, A. Machine Learning Predictions of Molecular Properties: Accurate Many-Body Potentials and Nonlocality in Chemical Space. J. Phys. Chem. Lett. 2015, 6, 2326-2331.

(13) Ramakrishnan, R.; von Lilienfeld, O. A. Many Molecular Properties from One Kernel in Chemical Space. chimia 2015, 69, 182-186.

(14) Rupp, M.; Tkatchenko, K.-R., Alexandre haand Müller; von Lilienfeld, O. A. Fast and Accurate Modeling of Molecular Atomization Energies with Machine Learning. Phys. Rev. Lett. 2012, 108, 058301.

(15) Barker, J.; Bulin, J.; Hamaekers, J.; Mathias, S. Localized Coulomb Descriptors for the Gaussian Approximation Potential. arXiv preprint arXiv:1611.05126 2016 ,

(16) von Lilienfeld, O. A.; Ramakrishnan, R.; Rupp, M.; Knoll, A. Fourier series of atomic radial distribution functions: A molecular fingerprint for machine learning models of quantum chemical properties. Int. J. Quantum 2015, 115, 1084-1093.

(17) Huan, T. D.; Mannodi-Kanakkithodi, A.; Ramprasad, R. Accelerated materials property predictions and design using motif-based fingerprints. Phys. Rev. B 2015, 92, 014106.

(18) Bartók, A. P.; Kondor, R.; Csányi, G. On representing chemical environments. Phys. Rev. B 2013, 87, 184115.

(19) De, S.; Bartók, A. P.; Csányi, G.; Ceriotti, M. Comparing molecules and solids across structural and alchemical space. 
Phys. Chem. Chem. Phys. 2016, 18, 13754-13769.

(20) Collins, C. R.; Gordon, G. J.; von Lilienfeld, O. A.; Yaron, D. J. Constant Size Molecular Descriptors For Use With Machine Learning. arXiv preprint arXiv:1701.06649 2016,

(21) Smith, J. S.; Isayev, O.; Roitberg, A. E. ANI-1: An extensible neural network potential with DFT accuracy at force field computational cost. Chem. Sci. 2017, 8, 3192-3203.

(22) Schütt, K. T.; Arbabzadah, F.; Chmiela, S.; Müller, K. R.; Tkatchenko, A. Quantum-chemical insights from deep tensor neural networks. Nat. Commun. 2017, 8, 13890.

(23) Dral, P. O.; von Lilienfeld, O. A.; Thiel, W. Machine Learning of Parameters for Accurate Semiempirical Quantum Chemical Calculations. J. Chem. Theory 2015, 11, 2120-2125.

(24) Weber, W.; Thiel, W. Orthogonalization corrections for semiempirical methods. Theor. Chem. Acc. 2000, 103, 495506.

(25) Dral, P. O.; Wu, X.; Spörkel, L.; Koslowski, A.; Thiel, W. Semiempirical Quantum-Chemical OrthogonalizationCorrected Methods: Benchmarks for Ground-State Properties. J. Chem. Theory 2016, 12, 1097-1120.

(26) Hansen, K.; Montavon, G.; Biegler, F.; Fazli, S.; Rupp, M.; Scheffler, M.; von Lilienfeld, O. A.; Tkatchenko, A.; Müller, K.R. Assessment and Validation of Machine Learning Methods for Predicting Molecular Atomization Energies. J. Chem. Theory Comput. 2013, 9, 3404-3419.

(27) Kearnes, S.; McCloskey, K.; Berndl, M.; Pande, V.; Riley, P. Molecular Graph Convolutions: Moving Beyond Fingerprints. J. Comput. Aided Mol. Des. 2016, 30, 595-608.
(28) Li, Y.; Tarlow, D.; Brockschmidt, M.; Zemel, R. Gated Graph Sequence Neural Networks. ICLR 2016,

(29) Curtiss, L. A.; Raghavachari, K.; Redfern, P. C.; Pople, J. A. Assessment of Gaussian-2 and density functional theories for the computation of enthalpies of formation. J. Chem. Phys. 1997, 106, 10631079 .

(30) Hickey, A. L.; Rowley, C. N. Benchmarking quantum chemical methods for the calculation of molecular dipole moments and polarizabilities. J. Phys. Chem. A 2014, 118, 3678-3687.

(31) Stowasser, R.; Hoffmann, R. What do the Kohn-Sham orbitals and eigenvalues mean? J. Am. Chem. Soc. 1999, 121, 3414 .

(32) Sinha, P.; Boesch, S. E.; Gu, C.; Wheeler, R. A.; Wilson, A. K. Harmonic Vibrational Frequencies: Scaling Factors for HF, B3LYP, and MP2 Methods in Combination with Correlation Consistent Basis Sets. J. Phys. Chem. A 2004, 108, 9213-9217.

(33) Geary, R. C. The Ratio of the Mean Deviation to the Standard Deviation as a Test of Normality. Biometrika 1935, 27, 310332 .

(34) DeTar, D. F. Calculation of Entropy and Heat Capacity of Organic Compounds in the Gas Phase. Evaluation of a Consistent Method without Adjustable Parameters. Applications to Hydrocarbons. J. Phys. Chem. A 2007, 111, 4464-4477.

(35) Huo, H.; Rupp, M. Unified Representation for Machine Learning of Molecules and Crystals. arXiv preprint arXiv:1704.06439 2017 ,

(36) Bartok, A. P.; De, S.; Poelking, C.; Bernstein, N.; Kermode, J.; Csanyi, G.; Ceriotti, M. Machine Learning Unifies the Modelling of Materials and Molecules. arXiv preprint arXiv:1706.00179 2017, 
(37) Rappe, A. K.; Casewit, C. J.; Colwell, K. S.; III, W. A. G.; Skiff, W. M. UFF, a full periodic table force field for molecular mechanics and molecular dynamics simulations. J. Am. Chem. Soc. 1992, 114, 10024-10035.

(38) Rogers, D.; Hahn, M. Extendedconnectivity fingerprints. J. Chem. Inf. Model. 2010, 50, 742-754.

(39) Besnard, J.; Ruda, G. F.; Setola, V.; Abecassis, K.; Rodriguiz, R. M.; Huang, X.-P.; Norval, S.; Sassano, M. F.; Shin, A. I.; Webster, L. A.; Simeons, F. R. C.; Stojanovski, L.; Prat, A.; Seidah, N. G.; Constam, D. B.; Bickerton, G. R.; Read, K. D.; Wetsel, W. C.; Gilbert, I. H.; Roth, B. L.; Hopkins, A. L. Automated design of ligands to polypharmacological profiles. Nature 2012, 492, 215-220.

(40) Lounkine, E.; Keiser, M. J.; Whitebread, S.; Mikhailov, D.; Hamon, J.; Jenkins, J. L.; Lavan, P.; Weber, E.; Doak, A. K.; Côté, S.; Shoichet, B. K.; Urban, L. Large-scale prediction and testing of drug activity on side-effect targets. Nature 2012, 486, 361-367.

(41) Huigens III, R. W.; Morrison, K. C.; Hicklin, R. W.; Flood Jr, T. A.; Richter, M. F.; Hergenrother, P. J. A Ring Distortion Strategy to Construct Stereochemically Complex and Structurally Diverse Compounds from Natural Products. Nature chemistry 2013, 5, 195.

(42) Todeschini, R.; Consonni, V. Handbook of Molecular Descriptors; Wiley-VCH, Weinheim, 2009.

(43) Faulon, J.-L.; Visco, Jr., D. P.; Pophale, R. S. The Signature Molecular Descriptor. 1. Using Extended Valence Sequences in QSAR and QSPR Studies. J. Chem. Inf. Comp. Sci. 2003, 43, 707.

(44) Visco, J.; Pophale, R. S.; Rintoul, M. D.; Faulon, J. L. Developing a methodology for an inverse quantitative structure activity relationship using the signature molecular descriptor. J. Mol. Graph. Model. 2002, 20, 429-438.

(45) O’Boyle, N. M.; Banck, M.; James, C. A.; Morley, C.; Vandermeersch, T.; Hutchison, G. R. Open Babel: An open chemical toolbox. J. Cheminform. 2011, 3, 1-14.

(46) Landrum, G. RDKit: Open-source cheminformatics. http://www.rdkit.org 2014, 3, 2012.

(47) Faber, F. A.; Hutchison, L.; Huang, B.; Gilmer, J.; Schoenholz, S. S.; Dahl, G. E.; Vinyals, O.; Kearnes, S.; Riley, P. F.; von Lilienfeld, O. A. Fast machine learning models of electronic and energetic properties consistently reach approximation errors better than DFT accuracy. arXiv preprint arXiv:1702.05532 2017,

(48) Müller, K.-R.; Mika, S.; Rätsch, G.; Tsuda, K.; Schölkopf, B. An introduction to kernel-based learning algorithms. IEEE transactions on neural networks 2001, 12, 181-201.

(49) Schölkopf, B.; Smola, A. J. Learning with kernels: support vector machines, regularization, optimization, and beyond; MIT press, 2002.

(50) Vovk, V. In Empirical Inference: Festschrift in Honor of Vladimir $N$. Vapnik; Schölkopf, B., Luo, Z., Vovk, V., Eds.; Springer Berlin Heidelberg: Berlin, Heidelberg, 2013; pp 105-116.

(51) Hastie, T.; Tibshirani, R.; Friedman, J. The Elements of Statistical Learning: Data Mining, Inference, and Prediction, Second Edition, 2nd ed.; Springer: New York, 2011.

(52) Hoerl,; Arthur, E.; Kennard,; Robert, W. Ridge Regression Biased Estimation for Nonorthogonal Problems. Technometrics 2000, 80 . 
(53) Pedregosa, F.; Varoquaux, G.; Gramfort, A.; Michel, V.; Thirion, B.; Grisel, O.; Blondel, M.; Prettenhofer, P.; Weiss, R.; Dubourg, V.; Vanderplas, J.; Passos, A.; Cournapeau, D.; Brucher, M.; Perrot, M.; Duchesnay, E. Scikit-learn: Machine Learning in Python. J. Mach. Learn. Res. 2011, 12, 2825-2830.

(54) Neal, R. M. Bayesian Learning for Neural Networks; Springer-Verlag New York, Inc.: Secaucus, NJ, USA, 1996.

(55) Zou, H.; Hastie, T. Regularization and variable selection via the elastic net. $J . R$. Stat. Soc. Series. B Stat. Methodol. 2005, 67, 301-320.

(56) Breiman, L. Random forests. Machine learning 2001, 45, 5-32.

(57) Duvenaud, D. K.; Maclaurin, D.; Iparraguirre, J.; Bombarell, R.; Hirzel, T.; Aspuru-Guzik, A.; Adams, R. P. Convolutional Networks on Graphs for Learning Molecular Fingerprints. Advances in Neural Information Processing Systems. 2015; pp 2215-2223.

(58) Desautels, T.; Krause, A.; Burdick, J. W. Parallelizing Exploration-Exploitation Tradeoffs in Gaussian Process Bandit Optimization. J. Mach. Learn. Res. 2014, 15, 4053-4103.

(59) Google HyperTune. https://cloud.google.com/ml/ (accessed 2016).

(60) Tew, D. P.; Klopper, W.; Heckert, M.; Gauss, J. Basis Set Limit CCSD(T) Harmonic Vibrational Frequencies. J. Phys. Chem. A 2007, 111, 11242-11248.

(61) Müller, K.-R.; Finke, M.; Murata, N.; Schulten, K.; Amari, S. A numerical study on learning curves in stochastic multilayer feedforward networks. Neural Comput. 1996, 8, 1085-1106.

(62) Huang, B.; von Lilienfeld, O. A. The "DNA" of chemistry: Scalable quantum machine learning with "amons". arXiv preprint arXiv:170\%.04146 2017,

(63) Gilmer, J.; Schoenholz, S. S.; Riley, P. F.; Vinyals, O.; Dahl, G. E. Neural Message Passing for Quantum Chemistry. Proceedings of the 34nd International Conference on Machine Learning, ICML 2017. 2017.

(64) Kirkpatrick, P.; Ellis, C. Chemical space. Nature 2004, 432, 823. 


\section{Log Error}

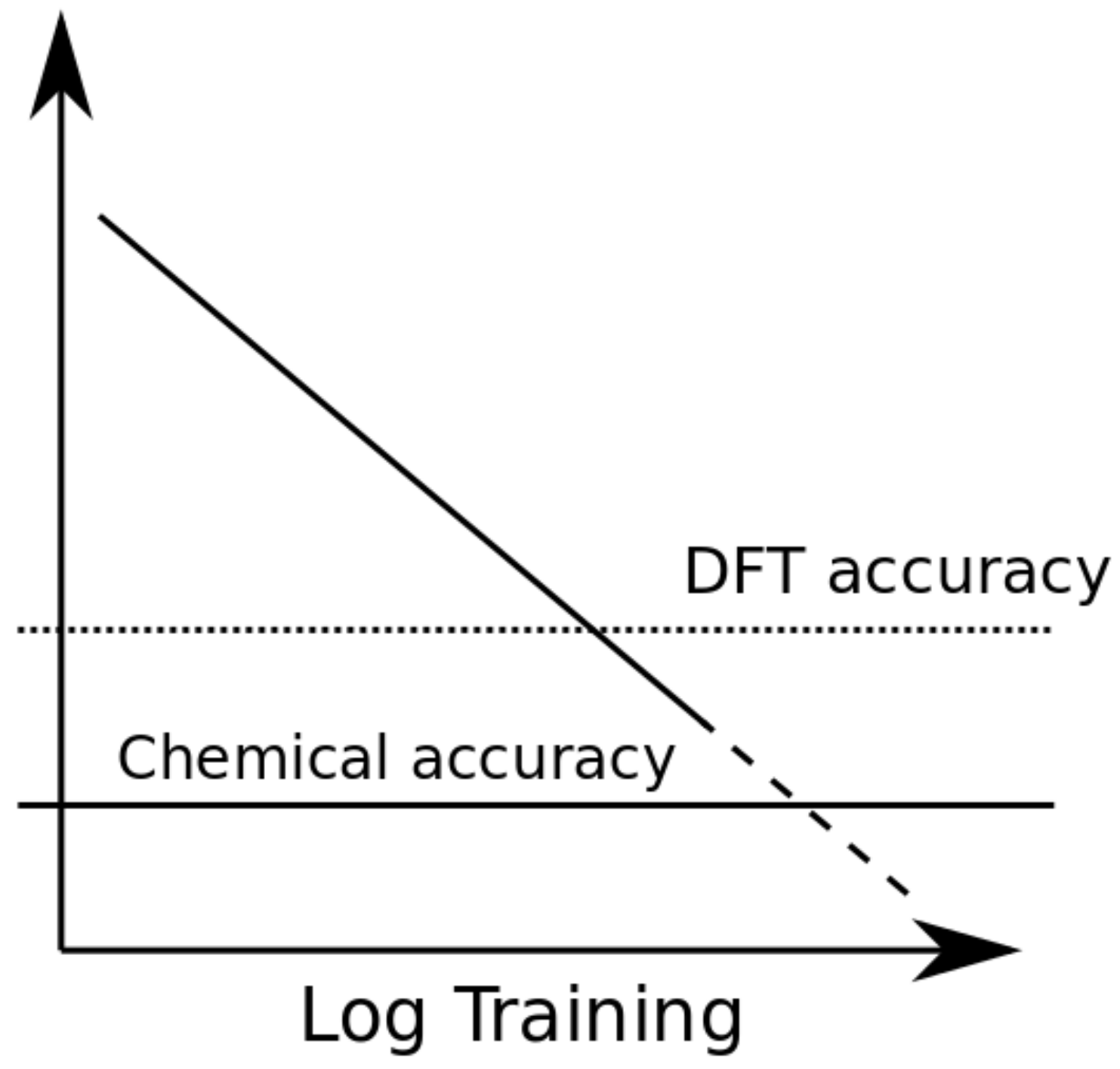

Figure 3: TOC 